\title{
The Accuracy of Shear Wave Elastography in the Assessment of Placental Invasion in Women with Placenta Previa
}

\author{
Original \\ Article \\ Mohamed A. Abdel-Hafeez, Sabry S. Hasan, Fatma F. Eljazwi, Ahmed M. Mansour \\ Department of Obstetrics and Gynecology, Faculty of Medicine, Ain-Shams University,Cairo, \\ Egypt
}

\begin{abstract}
Background: Placenta accreta spectrum is a term which describes abnormal invasion of the placenta into the uterine myometrium. The first line in diagnosis is ultrasonography and magnetic resonance imaging may complement the diagnosis. Aim: The aim of the work is to assess the accuracy of shear wave elastography in predication of placental invasion in women with placental previa.

Materials and Methods: This is a prospective cohort study which was conducted in Ain Shams University on 50 women with a diagnosis of anterior placenta previa and history of previous cesarean section who were pregnant at 32 weeks or more and were planned for delivery by elective cesarean section. They were subjected to ultrasonography and elastography study and the diagnosis of placenta accreta spectrum was confirmed/ excluded intraoperatively.

Results: Placental separation was normal in 36\% of cases, delayed in $46 \%$ of cases, partial in $6 \%$ of cases only whereas non separation occurred in $12 \%$ of cases. elastrgraphy had a sensitivity of $73.7 \%$, specificity of $90.3 \%$, positive predicting value of $82.4 \%$ and negative predictive value of $84.8 \%$ in diagnosing cases with abnormal placental invasion while sensitivity, specificity positive and negative predictive value of ultrasonography in diagnosing abnormal placental invasion was $89.5 \%, 96.8 \%, 94.4 \%$ and $93.8 \%$ respectively.

Conclusion: Elastography is sensitive and specific but inferior to ultrasonography in evaluating women with suspected placenta accreta spectrum but it may be useful in diagnosis of focal invasion.
\end{abstract}

Key Words: PAS, placenta accreta, placenta accreta spectrum, placental invasion, placenta previa, shear wave elastography

Received: 03 August 2021, Accepted: 18 August 2021

Corresponding Author: Mohamed A. Abd El-Hafeez, Department of Obstetrics and Gynecology, Faculty of Medicine, Ain-Shams University, Cairo, Egypt, Tel.: +20 1069491030, E-mail: abdelhafeezmohamed014@gmail.com

ISSN: 2090-7265, August 2021, Vol.11, No. 3

\section{INTRODUCTION}

Placenta accreta was originally described by Irving and Hertig in 1937 as an abnormal adherence of the afterbirth. The term was also widely used to refer to abnormal placental invasion which was not included in the original definition. Abnormally implanted placenta is classified according to depth of invasion into accreta, increta and percreta or according to the area attached to the myometrium into total, partial or focal. ${ }^{[1]}$

Recently, the term placenta accreta spectrum (PAS) is used to describe the whole scope from morbidly adherence to placenta percreta ${ }^{[2,3]}$. The major risk factors for PAS are history of previous cesarean delivery and placenta previa in the current pregnancy ${ }^{[2-4]}$. The pathogenesis of PAS is believed to results from defective decidua basalis with abnormal invasion of the placental tissue ${ }^{[5]}$.

PAS is diagnosed by different imaging modalities and ultrasound scan is still the first line investigation for diagnosing abnormal placental invasion subgroup ${ }^{[3]}$. Magnetic resonance imaging may complement sonography as it is more accurate to assess the depth of invasion but otherwise ultrasonography remains the primary tool for diagnosis ${ }^{[6]}$. Different features with different accuracy have been reported for diagnosing PAS with ultrasound as well as with MRI ${ }^{[6,7]}$.

Elastography is a relatively new modality applied during an ultrasound scan and generates information regarding the elastic properties of the scanned tissue ${ }^{[8]}$. This hypothesis was actually derived from basic clinical examination as palpation is applied to detect lumps which usually represent cancerous tissue which has stiffer than the adjacent normal tissue ${ }^{[9]}$. The concept of elastography is that the generated information about the degree of softness of the tissue may reflect the presence or absence of a certain disease $\mathrm{e}^{[10]}$.

Several applications for elastography in medicine have been reported and in obstetrics it was first used to evaluate the consistency of the cervix in the setting of preterm labor or induction of labor ${ }^{[5]}$. Research was then directed towards evaluation of elastography in various placental pathological conditions. Elastography studies have demonstrated 
harder placental tissue in women with pregnancy induced hypertension as well as in growth restricted fetuses ${ }^{[9,11]}$. The aim of the current study is to assess the accuracy of shear wave elastography in predicating placental invasion in women with placental previa.

\section{PATIENTS AND METHODS}

This study was a prospective cohort observational study which was conducted at the department of Obstetrics and Gynecology, Ain-Shams University Maternity Hospital between May 2018 and May 2019. The study was approved by the ethical committee of Ain Shams University. Verbal consent was taken from enrolled patients after explaining the purpose of the examination.

The study included 50 women with a diagnosis of placenta previa anterior, gestational age of 32 weeks or more and a history of at least one previous cesarean section delivery who have been decided to undergo elective cesarean section delivery.

Women with high blood pressure, preeclampsia, gestational diabetes mellitus or multiple gestations were excluded from the study.

Gestational age was confirmed by an ultrasonography report performed in early pregnancy. Diagnosis of placenta previa was made during a routine midpregnancy scan and it was confirmed by a follow up transvaginal sonographic examination carried out at 32 weeks gestation to confirm persistence of placenta previa.

After inclusion, all women were subjected to Doppler study on placental bed and shear wave elastography and then they were followed up at obstetrics outpatient clinic until delivery and the decision for timing of delivery was individualized for each woman. The final diagnosis of PAS was confirmed during surgery.

Sonographic examination involved standard fetal biometry, anomaly scanning and placental localization, thickness, and morphology and the recommended criteria were applied to confirm the diagnosis of $\mathrm{PAS}^{[3]}$. The sonographic findings considered as criteria of PAS were vascular lacunae, irregular retroplacental sonoluscent zone, loss of retroplacental "clear space" and interruption of bladder line. The color Doppler criteria suggestive of PAS that was assessed included the following; diffuse or focal intraparaenchymal lacunar flow, vascular lakes with turbulent flow, hypervascularity of serosa-bladder interface and prominent subplacentalvenous complex.

A shear wave capable device supported with 1-6 MHz curved array transducer (Super Sonic Imagine, Aix en Provence, France) was used to perform sonoelastography examination which was carried out in the supine position, images were obtained in sagittal planes with a perpendicular view of the placenta and women were asked to breathe slightly before image acquisition. Images were displayed as an overlay in dual mode with gray-scale images simultaneously. A rectangular adjustable electronic box was used for shear wave elastography (SWE) examinations in the elastogram screen and the placental tissue stiffness was displayed in the adjustable box (Figures 1-3).

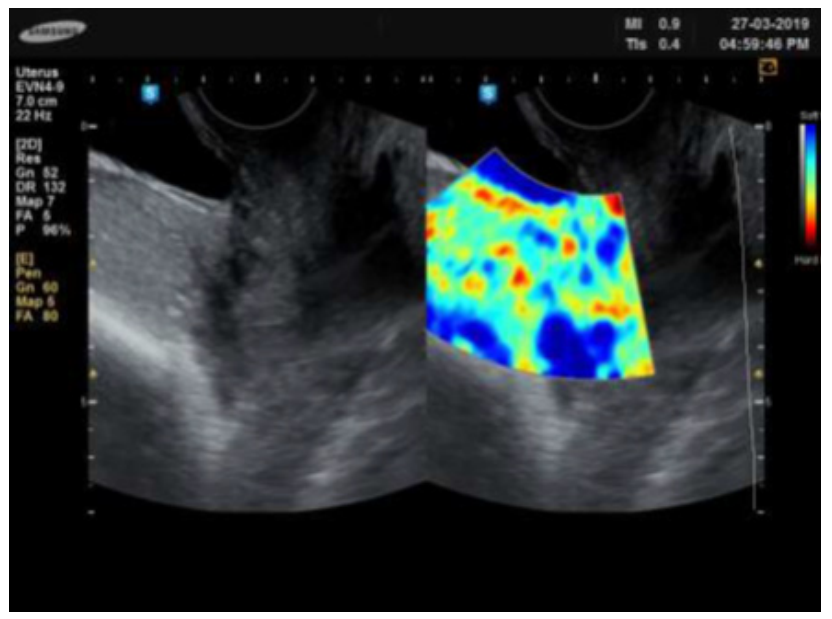

Fig. 1: A case in which sonography and elastography showed no invasion which was confirmed intraoperatively

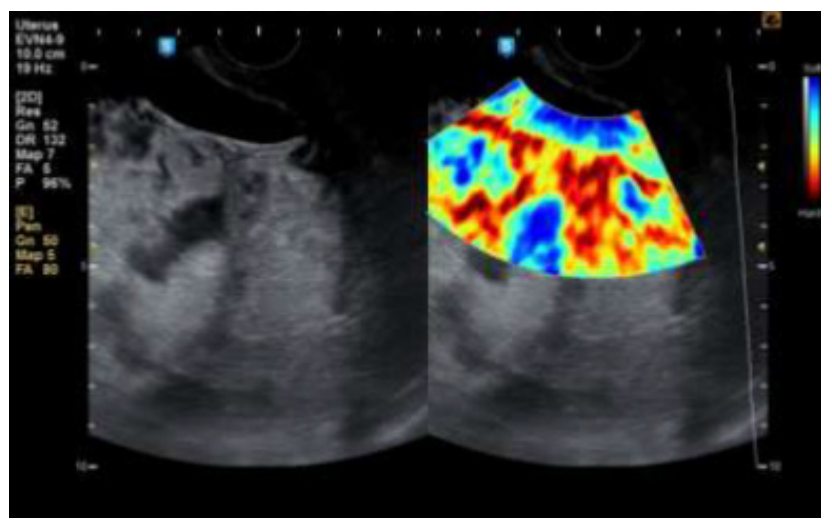

Fig. 2: A case in which sonography suggested placental invasion (left image) and elastography showed tissue hardness demonstrated by the red color (right image) with confirmed intraoperative placental invasion

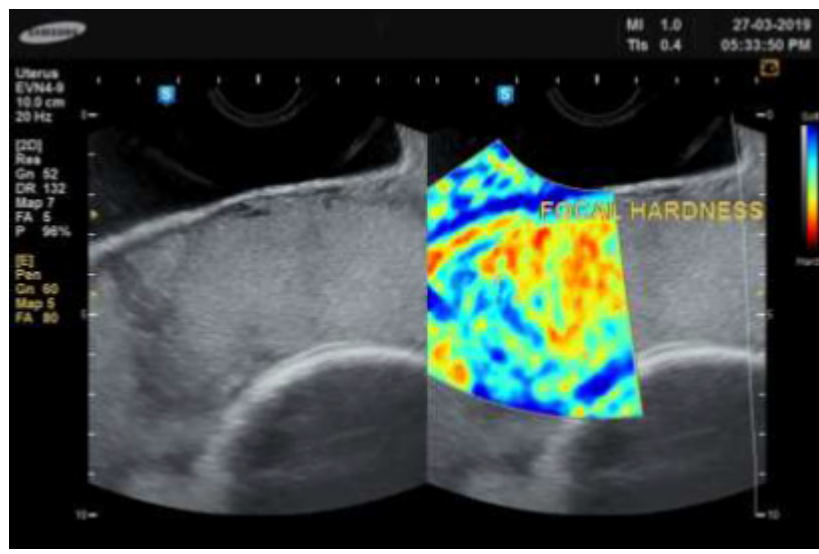

Fig. 3: A case in which sonography showed no signs of placental invasion (left image) but elastography showed focal tissue hardness with confirmed intraoperative focal placental invasion 
Elastograms were obtained from central and peripheral parts of the placenta and region of interest (ROI, QBOX) tool was fixed to $5 \mathrm{~mm}$ diameter placed on the stiffest area on the color map which would be the most dark colored area and represents the most suspected zone for placental invasion, only one ROI was used for each central and peripheral parts of the placenta. Shear modulus data (in kilopascals, kPA) have been automatically displayed for all ROI.

Delivery was planned by an elective cesarean section and was carried out by the lecturer on duty and the surgeon was not informed of the elastography result and was asked to comment on placental separation. PAS was confirmed by intraoperative data with confirmed diagnosis if 1) delayed or non separation of the placenta and /or 2) any surgical intervention required specifically for managing cases with PAS.

\section{RESULTS}

The mean of age of the included women was $29.4 \pm 2.6$ years, the mean of BMI was $28.5 \pm 2.7 \mathrm{Kg} / \mathrm{m}^{2}$ and a median of 3 previous cesarean section delivery. The mean of intraoperative blood loss was 1070.0 \pm 522.6 $\mathrm{ml}$. Regarding placental separation, it was delayed in $23(46 \%)$ patients, partial separation in $3(6 \%)$ patients and no separation occurred in $6(12 \%)$ patients. Hysterectomy was performed in $12(24 \%)$ cases. Reanalysis of complications after categorizing the patients into 2 subgroups: placental invasion and no invasion showed that as expected the intraoperative blood loss, the incidence of severe bleeding, blood transfusion and hysterectomy were significantly more frequent in the subgroup with confirmed placental invasion.

Using intraoperative data as the reference test to confirm PAS diagnosis, PAS was confirmed in $19(38 \%)$ patients, while a suspected PAS was reported in $17(34 \%)$ patients using elastography and in $18(36 \%)$ patients using ultrasonography. As regards elastography performance, true positive cases were $28 \%$, true negative were $56 \%$, false positive cases were $6 \%$ while false negative cases were $10 \%$. This resulted in a sensitivity of $73.7 \%$, a specificity of $90.3 \%$, positive predictive value (PPV) of $82.4 \%$ and negative predictive value (NPV) of $84.8 \%$. While performance of ultrasonography showed true positive cases in $4 \%$, true negative cases in $60 \%$, false positive cases in $2 \%$ and false negative cases in $4 \%$. This resulted in a sensitivity of $89.5 \%$, a specificity of $96.8 \%$, PPV and NPV of $94.4 \%$ and $93.8 \%$ respectively. Figures 1-3 demonstrate ultrasonography and elastography images of 3 of the included patients.

As shown in Table 1, there was a substantial agreement between diagnosing PAS by ultrasonography and elastography (kappa $=0.78)$. confirmed PAS by intraoperative findings showed a perfect agreement with ultrasonography (kappa $=0.87$ ) while elastography showed a substantial agreement with confirmed PAS $($ kappa $=0.65)($ Table 2).
Our results demonstrate that ultrasonography has higher diagnostic characteristics than elastography in the diagnosis of placental invasion using intraoperative confirmation as the gold standard for diagnosis (Table 3).

\begin{tabular}{ccccc}
\hline & & \multicolumn{2}{c}{ ultrasonography } & \multirow{2}{*}{ kappa } \\
\cline { 3 - 4 } & & invasion & no invasion & \\
\hline \multirow{2}{*}{ elastography } & invasion & $15(30)$ & $2(4)$ & 0.780 \\
& no invasion & $3(6)$ & $30(60)$ & $(0.562-0.998)$ \\
\hline
\end{tabular}

Data presented as number (percentage), analysis done using Kappa test and kappa is reported as value and its $95 \%$ confidence interval

Table 2: Agreement of intraoperative diagnosis of placental invasion with elastography and with ultrasonography

\begin{tabular}{ccccc}
\hline & & \multicolumn{2}{c}{ intraoperative } & \multirow{2}{*}{ kappa } \\
\cline { 2 - 4 } & & invasion & no invasion & \\
\hline \multirow{3}{*}{ elastography } & invasion & $14(28)$ & $3(6)$ & 0.653 \\
& no & $5(10)$ & $28(56)$ & $(0.435-0.872)$ \\
& invasion & & & \\
invasion & $17(34)$ & $1(2)$ & 0.871 \\
ultrasonography & no & $2(4)$ & $30(60)$ & $(0.730-1.000)$ \\
& invasion & $2(4)$ & & \\
\hline
\end{tabular}

Data presented as number (percentage), analysis done using Kappa test and kappa is reported as value and its $95 \%$ confidence interval

Table 3: diagnostic characteristics of elastography and ultrasonography in diagnosis of placental invasion

\begin{tabular}{lcc}
\hline Character & elastography & ultrasonography \\
\hline Sensitivity & $73.7 \%$ & $89.5 \%$ \\
Specificity & $90.3 \%$ & $96.8 \%$ \\
diagnostic accuracy & $84.0 \%$ & $94.0 \%$ \\
Youden's index & $64.0 \%$ & $86.2 \%$ \\
Positive Predictive value & $82.4 \%$ & $94.4 \%$ \\
Negative Predictive value & $84.8 \%$ & $93.8 \%$ \\
Positive likelihood ratio (LR+) & 7.61 & 27.74 \\
Negative likelihood ratio (LR-) & 0.29 & 0.11 \\
Diagnostic odd ratio (LR) & 26.13 & 255.00 \\
\hline
\end{tabular}

\section{DISCUSSION}

The technology of elasticity imaging has been applied in clinical practice over the past decade. Clinical benefits of elastography have been shown in imaging of the breas ${ }^{[12]}$, thyroid $^{[13]}$ and liver ${ }^{[14]}$ with accumulating evidence of improved interpretation specially for classifying breast lesions ${ }^{[12]}$. It is hypothesized that if the value demonstrated with this modality is extrapolated to assessment of various tissue invasiveness, it may be implicated in evaluating women with suspected abnormal placental invasion. Studies evaluating the role of elastography in the workup of PAS are scarce ${ }^{[11,15]}$.

The current study shows that significantly stiffer placental tissue is found in cases with PAS compared to normally implanted placentas. There was substantial 
agreement between elastography and intraoperative confirmed PAS. We searched the literature for studies evaluating the role of elastography in diagnosis of placental invasion and only 3 studies could be retrieved ${ }^{[16-18]}$.

In line with this result, Davie et al ${ }^{[16]}$ reported on a case with PAS who presented to them with a history of previous 2 cesarean section and a history of retained placenta in the antecedent pregnancy with confirmed PAS by histopathological examination of hysteroscopic curettage specimen. For the management of their case, she was subjected to ultrasonography, elastography and MRI imaging studies and all reported a diagnosis of PAS. Finally, the woman underwent an urgent hysterectomy in toto for interactive bleeding at around midpregnancy and PAS was confirmed by histopathological study.

Also in agreement with our results, Cim et al ${ }^{[17]}$ found elastography scores to be significantly higher in the group with placental invasion and suggested that it may be helpful in predicting abnormal placental invasion. Tissue stiffness was found in all the studied areas (central and peripheral placenta), this observation supports previous results which reported that placental elasticity may be represented by elastography imaging of the placental center as well as the placental periphery ${ }^{[15]}$.

These results partially agreed with a previous study evaluating elastography in the context of $\mathrm{PAS}^{[18]}$. That case control study compared women with placenta previa anterior to women with normal placental location and reported that SWE is significantly higher in cases with placenta previa compared to placenta with normal location and in agreement with current results, this result was demonstrated in all the studied areas but contrary to our results, elasticity values were comparable for cases of placenta previa with and without abnormal placenta invasion $^{[18]}$

In the present study, ultrasonography had higher diagnostic characteristics than elastography in the diagnosis of PAS. However, there was statistically significant high agreement between placental invasion diagnoses by ultrasonography and elastography.

Ultrasonography is still recommended as first line diagnostic test for PAS ${ }^{[2,3,6]}$ and it has been reported that ultrasonography has a high sensitivity and specificity in diagnosing PAS ${ }^{[19]}$. This is in line with the current results which show that ultrasonography has a sensitivity of $89.5 \%$ and a specificity of $96.8 \%$.

\section{CONCLUSION}

Elastography is sensitive and specific but inferior to ultrasonography in evaluating women with suspected placenta accreta spectrum and it may be useful in diagnosis of focal invasion.

\section{CONFLICT OF INTERESTS}

There are no conflicts of interests.

\section{REFERENCES}

1. Eller AG, Bennett MA, Sharshiner M, Masheter C, Soisson AP, Dodson M, Silver R. Maternal morbidity in case of placenta accreta managed by a multidisciplinary care team compared with standard obstetric care. Obstet Gynecol 2011; 117: 331-337.

2. Jauniaux E, Ayres-de-campos D, Langhoff-Roos J et al. FIGO classification for the clinical diagnosis of placenta accreta spectrum disorders. Int J Gynecool Obstet 2019; 146(1): 20-24. doi: 10.1002/ijgo.12761

3. Jauniaux ERM, Alfirevic Z, Bhide AG, Belfort MA, Burton GJ, Collins SL, Dornan S, Jurkovic D, Kayem G, Kingdom J, Silver R, Sentilhes L on behalf of the Royal College of Obstetricians and Gynaecologists. Placenta Praevia and Placenta Accreta: Diagnosis and Management. Green-top Guideline No. 27a. BJOG 2018. doi: 10.1111/1471-0528.15306.

4. Panaiotova, J., Tokunaka, M., Krajewska, K., Zosmer, N., and Nicolaides, K. H. Screening for morbidly adherent placenta in early pregnancy. Ultrasound in Obstetrics \& Gynecology 2019, 53(1), 101-106.

5. Swiatkowska-Freund M, Traczyk-Łoś A, Preis K, Łukaszuk M, Zielińska K. Prognostic value of elastography in predicting premature delivery. Ginekol Pol 2014; 85: 204-7.

6. Jha P, Poder L, Bourgioti C, et al. Society of Abdominal Radiology (SAR) and European Society of Urogenital Radiology (ESUR) joint consensus statement for MR imaging of placenta accreta spectrum disorders. Eur Radiol 2020; 30(5): 2604-2615.

7. Berkley EM, andAbuhamad AZ. The prenatal diagnosis of placenta accreta, is ultrasound all we need? J Ultrasound Med. 2013; 32: 1345-1350.

8. Sporea I. clinical elastography. Med Ultrason 2018; 20(3): 263-264.

9. Bamber J, Cosgrove D, Dietrich CF, et al. EFSUMB guidelines and recommendations on the clinical use of ultrasound elastography. Part 1: basic principles and technology. Ultraschall Med 2013; 34:169-184.

10. Wells PN, Liang HD. Medical ultrasound: imaging of soft tissue strain and elasticity. J R Soc Interface 2011; 1521-1549. Doi: 10.1098/rsif.2011.0054.

11. Kilic F, Kayadibi Y, Yuksel MA, et al. Shear wave elastography of placenta: in vivo quantitation of placental elasticity in preeclampsia. Diagn Interv Radiol. 2015; 21:202-207. 
12. Carlsen JF, Hansen KL, Ewertsen C, Nielsen MB. Elastography in breast imaging. Elastografie Brustbildgebung. Ultraschall Med 2019; 40 (6): 688-691.

13. Cosgrove $\mathrm{D}$, Barr $\mathrm{R}$, Bojunga $\mathrm{J}$ et al. WFUMB Guidelines and Recommendations on the clinical of ultrasound elastography: part 4. Thyroid. Ultrasound Med Biol 2017; 43(1): 4-26.

14. Jung, Kyu Sik; Kim, and Seung Up."Clinical applications of transient elastography" Clinical and Molecular Hepatology 2012; 18 (2): 163-73.

15. Li WJ, Wei ZT, Yan RL, et al. Detection of placenta elasticity modulus by quantitative real-time shear wave imaging. Clin Exp Obstet Gynecol. $2012 ; 39: 470-473$.
16. Davie S, Wong WL, Clapham T, et al. case Rep Obstet Gynecol 2016; 2016: 4909431. Doi: $10.1155 / 2016 / 4909431$.

17. Cim N, Tolunay HE, Boza B et al. use of ARFI elastography in the prediction of placental invasion anomaly via a new Virtual Touch Quantification Technique. J Obstet Gynecol 2018; 38 (7): 911-915.

18. Davutoglu AE, Ariöz Habibi H, Ozel A, Yuksel MA, et al. The role of shear wave elastography in the assessment of placenta previa-accreta, J Matern Fetal Neonatal Med 2018; 31(12): 1660-1662. Doi: $10.1080 / 14767058.2017 .1322059$

19. D'Antonio F, Iacovella C, and Bhide A. Prenatal identification of invasive placentation using ultrasound: systematic review and meta-analysis. Ultrasound Obstet Gynecol 2013; 42:509-517. 